\title{
SCAPE: Safe Charging with Adjustable PowEr
}

\author{
Haipeng Dai*, Yunhuai Liu ${ }^{\dagger}$, Guihai Chen*‡, Xiaobing Wu*, Tian $\mathrm{He}^{\S}$ \\ *State Key Laboratory for Novel Software Technology, Nanjing University, Nanjing, Jiangsu 210023, CHINA \\ $\dagger$ Third Research Institute of Ministry of Public Security, Shanghai, CHINA \\ ${ }^{\ddagger}$ Shanghai Key Laboratory of Scalable Computing and Systems, Shanghai Jiao Tong University, Shanghai 200240, CHINA \\ ${ }^{\S}$ Computer Science and Engineering, University of Minnesota, Minneapolis, MN 55455, USA \\ Emails: \{dhpphd2003, yunhuai.liu\}@gmail.com, \{gchen, wuxb\}@nju.edu.cn, tianhe@cs.umn.edu
}

\begin{abstract}
Wireless power transfer technology is considered as one of the promising solutions to address the energy limitation problems for end-devices, but its incurred potential risk of electromagnetic radiation (EMR) exposure is largely overlooked by most existing works. In this paper, we consider the Safe Charging with Adjustable PowEr (SCAPE) problem, namely, how to adjust the power of chargers to maximize the charging utility of devices, while assuring that EMR intensity at any location in the field does not exceed a given threshold $R_{t}$. We present novel techniques to reformulate SCAPE into a traditional linear programming problem, and then remove its redundant constraints as much as possible to reduce computational effort. Next, we propose a distributed algorithm with provable approximation ratio $(1-\epsilon)$. Through extensive simulation and testbed experiments, we demonstrate that our $(1-\epsilon)$-approximation algorithm outperforms the Set-Cover algorithm by up to $23 \%$, and has an average performance gain of $41.1 \%$ over the SCP algorithm in terms of the overall charging utility.
\end{abstract}

\section{INTRODUCTION}

In recent years, wireless power transfer technology [1] has been attracting great interests of industry and researchers. As a commercialized and controllable technology, it is one of the promising technologies to address the energy limitation problems for end-devices such as RFIDs [2], sensors [3], cell phones [4], laptops [5], vehicles [6] and unmanned planes [7].

Though there has emerged a variety of works dedicated to energy efficiency issues with respect to wireless power transfer technology [8]-[13], most of them overlooked the potential risk of electromagnetic radiation (EMR) brought by this technology. Exposure to high EMR, however, has been widely recognized as a threat to human health. Its potential risks include but not limited to mental diseases [14], tissue impairment [15] and brain tumor [16]. In addition, there has been solid evidence that pregnant women and children are even more vulnerable to high EMR exposure [17] [18]. For example, Gandhi et al. [18] found that children's heads absorb over two times of RF than adults, and their absorption of the skull's bone marrow can be ten times greater than adults. These facts suggest the need for considering EMR safety when applying wireless power transfer technology.

In this paper, we attempt to improve the overall charging performance under EMR safety concern, where chargers can continuously adjust their power level within an appropriate range. Basically, our objective is to maximize the overall charging utility of devices by adjusting the power of chargers, while assuring that no location has EMR intensity exceeding a given threshold.

Intuitively, this problem is quite challenging as the EMR safety requirement is imposed on every point in the field, which corresponds to an infinite number of constraints. To make the problem tractable, we present an approximation approach to reformulate the problem as a linear programming problem with limited constraints, and also devise a novel distributed approach to reduce the computational efforts of the problem. After that, we develop a $(1-\epsilon)$-approximation distributed algorithm to deal with this problem.

The main contributions of this paper are listed as follows.

- To the best of our knowledge, this is the first paper considering the problem of maximizing the charging efficiency of the network under EMR safety concern, by adjusting the power of chargers. We formulate this problem as Safe Charging with Adjustable PowEr (SCAPE) problem.

- We present an area discretization technique to help reformulating the problem into a traditional linear programming (LP) problem. Further, we propose a novel distributed redundant constraint reduction scheme to cut down the number of constraints, and thus reduce the computational efforts of the LP problem.

- We develop a distributed algorithm to deal with the SCAPE problem, and prove that it achieves $(1-\epsilon)$ approximation ratio.

- We build a testbed to evaluate the performance of our algorithms. Experimental results show that our algorithm successfully controls the maximal EMR in the field under a given threshold $R_{t}$, and has an average performance gain of $41.1 \%$ compared with the SCP algorithm. Furthermore, we also conduct comprehensive simulations. The results show that our algorithm outperforms the Set-Cover algorithm by up to $23 \%$.

The remainder of the paper is organized as follows. We review related work in Sec. II, and formally define the problem in Sec. III. Sec. IV introduces a novel approach to reformulate the problem, and Sec. V proposes a distributed method to reduce its computational efforts. Next, we present an approximation algorithm in Sec. VI. Sec. VII and Sec. VIII present extensive simulation results and testbed experiment results to validate our theoretical fingings, and Sec. IX concludes. 


\section{RELATED WORK}

In this section, we briefly review related works studying energy efficiency problems in wireless rechargeable sensor networks with wireless power transfer technology, and that considered EMR safety.

First, we concentrate on the works about how to deploy static chargers to maximize the charging efficiency of sensor nodes. For example, He et al. [8] considered the deployment problem of chargers such that static or mobile rechargeable tags can receive sufficient power to keep continuous operation, while the required number of chargers can be minimized. Dai et al. [9] [13] further improved the solution by taking into consideration practical issues such as battery constraints of tags. In [10], Chiu et al. studied the problem of maximizing the survival rate of end-devices with prior knowledge of the mobility model of sensor nodes. Liao et al. [11] adopted a more practical charging model by assuming that the coverage area of a charger is a cone, and considered the placement problem of chargers in three-dimensional space. Their objective is to minimize the number of deployed chargers while assuring that all sensor nodes are covered.

All the above schemes did not consider EMR safety during charging process. [19] is the first and only work, as far as we know, to study the energy efficiency problem under concern of EMR safety. We emphasize that this work is fundamentally different from that of [19] in the following aspects. First of all, [19] considered a simplified charger scheduling model in which chargers can be only in either of the on/off states, while we assume that the power of chargers is adjustable in this paper. Second, the proposed algorithm in [19] is essentially a centralized algorithm. In contrast, the algorithm presented in this paper is a distributed one. Third, though the algorithm in [19] has been proved to outperform the optimal solution for the problem with a relaxed EMR threshold $(1-\epsilon) R_{t}$, it is, however, not an approximation algorithm since it relaxes the EMR constraints. Conversely, our distributed algorithm provably achieves an approximation ratio of $(1-\epsilon)$.

\section{Problem Statement}

\section{A. Preliminaries}

Suppose that there is a set of $n$ identical stationary wireless power chargers $S=\left\{s_{1}, s_{2}, \ldots, s_{n}\right\}$ and $m$ rechargeable devices $O=\left\{o_{1}, o_{2}, \ldots, o_{m}\right\}$ distributed on a two-dimensional plane. The devices can harvest wireless power originated from the chargers and thus maintain normal working.

We assume that all the chargers can continuously adjust its power level from 0 to a maximum power. When a charger works at the maximum power, the received power $P(d)$ by a device with a distance $d$ from the charger can be quantified by an empirical model [8], i.e.,

$$
P(d)= \begin{cases}\frac{\alpha}{(d+\beta)^{2}}, & d \leq D \\ 0, & d>D\end{cases}
$$

where $\alpha$ and $\beta$ are known constants determined by the hardware of the charger and the receiver, as well as the environment. Because of the hardware constraint, the received power from the charger decreases dramatically as the distance increases, and the energy field far away from the charger will be too small to be received by a node. We characterize this property by using $D$ to denote the farthest distance a charger can reach, as Eq. 1 illustrates.

We define adjusting factor $x_{i}\left(0 \leq x_{i} \leq 1, i=1, \ldots, n\right)$ as the ratio of the current adjusted power to the maximum allowed power for the charger $s_{i}$. Therefore, the power a device received from a charger with distance $d$ and adjusting factor $x_{i}$ can be expressed as $P(d) x_{i}$. Besides, we assume the wireless power originating from multiple chargers received by a receiver is additive [8].

We assume that each charger is aware of its location. Two chargers are neighbors to each other if and only if their coverage areas intersect. Formally, we denote by $N\left(s_{i}\right)$ the set of neighbors of the charger $s_{i}$. Each charger can simultaneously communicate with their neighbors wirelessly during charging process [20] [21], which implies that the wireless communication range is at least twice the charging range, i.e., $2 D$. This assumption is practical since the effective charging distance for most off-the-shelf products is usually short, e.g., less than $10 \mathrm{~m}$ for TX91501 power transmitters produced by Powercast [3], while the wireless communication range for chargers is typically larger than $20 \mathrm{~m}$.

For the charging utility model, we define the charging utility to be proportional to the charging power, namely

$$
u\left(o_{j}\right)=C_{1} \sum_{i=1}^{n} P\left(d\left(s_{i}, o_{j}\right)\right) x_{i},
$$

where $d\left(s_{i}, o_{j}\right)$ is the distance from the charger $s_{i}$ to the device $o_{j}$, and $C_{1}$ is a predetermined constant.

We adopt the EMR model which is proposed and experimentally verified by [19]. That is, the intensity of EMR is proportional to the received power there, i.e., $e(d)=$ $C_{2} P(d) x_{i}$ where $d$ is the distance and $C_{2}$ is the constant to capture the linear relation. Assuming EMR is also additive, the accumulated EMR at a location $p$ is thus

$$
e(p)=\sum_{s_{i} \in S} e\left(d\left(s_{i}, p\right)\right)=C_{2} \sum_{s_{i} \in S} P\left(d\left(s_{i}, p\right)\right) x_{i} .
$$

A summary of the notations in this paper is given in Table I.

\section{B. Problem Description}

With the aforementioned models, we describe and mathematically formulate our problem in this subsection.

In order to control the EMR level over the field, we establish an appropriate EMR threshold $R_{t}$ and require that EMR at any point $p$ in the field should not exceed $R_{t}$. By Eq. 3, this requirement can be formally expressed as

$$
\forall p \in \mathbb{R}^{2}, \quad C_{2} \sum_{i=1}^{n} P\left(d\left(s_{i}, p\right)\right) x_{i} \leq R_{t} .
$$


TABLE I

NOTATIONS

\begin{tabular}{r|l}
\hline Symbol & Meaning \\
\hline \hline$s_{i}, S$ & Charger $i$, charger set \\
$o_{j}, O$ & Device $j$, device set \\
$P(d)$ & Received power from distance $d$ \\
$D$ & Farthest distance a charger can reach \\
$d\left(s_{i}, o_{j}\right)$ & Distance from charger $s_{i}$ to device $o_{j}$ \\
$d\left(s_{i}, p\right)$ & Distance from charger $s_{i}$ to point $p$ \\
$u\left(o_{j}\right)$ & Charging utility of device $o_{j}$ \\
$e(d), e(p)$ & EMR from distance $d$, EMR at point $p$ \\
$x_{i}$ & Adjusting factor of charger $s_{i}$ \\
$R_{t}$ & Hard threshold of EMR safety \\
$N\left(s_{i}\right)$ & Neighbor set ${ }^{1}$ of charger $s_{i}$ \\
\hline
\end{tabular}

1 Two chargers are neighbors to each other if and only if their coverage areas intersect.

SCAPE $\rightarrow \begin{gathered}\text { Sec. IV } \\ \begin{array}{l}\text { Area Discretization and } \\ \text { Problem Reformulation }\end{array}\end{gathered} \longrightarrow \begin{gathered}\text { Sec. V } \\ \text { Distributed Redundant } \\ \text { Constraint Reduction }\end{gathered} \longrightarrow \begin{gathered}\text { Sec. VI } \\ \text { Distributed Algorithm }\end{gathered}$

Fig. 1. Illustration of SCAPE workflow

On the other hand, our objective is to maximize the overall charging utility from all devices, namely, $\sum_{j=1}^{m} u\left(o_{j}\right)$. By Eq. 2, we have

$$
\sum_{j=1}^{m} u\left(o_{j}\right)=C_{1} \sum_{i=1}^{n}\left(\sum_{j=1}^{m} P\left(d\left(s_{i}, o_{j}\right)\right)\right) x_{i} .
$$

To sum up, the Safe Charging with Adjustable PowEr problem (SCAPE) can thus be defined as follows

$$
\begin{aligned}
& \text { (P1) } \quad \max \quad C_{1} \sum_{i=1}^{n}\left(\sum_{j=1}^{m} P\left(d\left(s_{i}, o_{j}\right)\right)\right) x_{i} \\
& \text { s.t. } \quad \forall p \in \mathbb{R}^{2}, \quad C_{2} \sum_{i=1}^{n} P\left(d\left(s_{i}, p\right)\right) x_{i} \leq R_{t}, \\
& \\
& 0 \leq x_{i} \leq 1 \quad(i=1,2, \ldots, n) .
\end{aligned}
$$

It is very challenging to solve SCAPE seeing from the above formulation. The constraint in SCAPE is imposed on every point on the plane, which means that there is indeed an infinite number of constraints. We will introduce the overview of our solution to address this problem below.

\section{Overview of Our Solution}

To convey the main idea of the scheme in this paper, we first present an overview of our solution.

The workflow of our solution is illustrated in Fig. 1. First of all, in view of the challenge caused by the infinite number of constraints in problem P1, we propose a novel area discretization method to partition the field into a limited number of sub-areas. By expressing the EMR safety requirement in each of these sub-areas as a constraint, we reformulate the original intractable problem P1 into a classical linear programming problem (Sec. IV). Next, we discuss how to implement the area discretization method in a distributed manner. As the number of obtained linear constraints explodes with the network scale and the granularity of area discretization and leads to high computational efforts, we develop a distributed algorithm to identify and reduce the redundant constraints at the second step (Sec. V). Finally, we propose a distributed algorithm to address the problem, which provably achieves $(1-\epsilon)$-approximation ratio (Sec. VI).

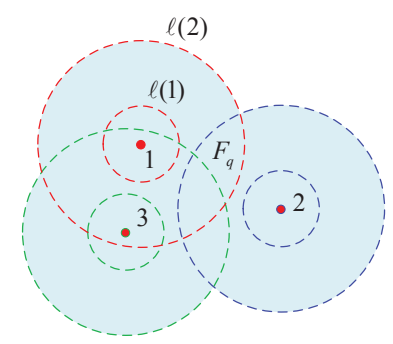

Fig. 2. Illustration of plane discretization

\section{Area Discretization and Problem REFORMULATION}

In this section, we first introduce an area discretization method to reduce the number of constraints from infinite to finite, and thereby reformulate our problem into a traditional linear programming problem. Finally, we discuss how to implement this method in a distributed manner.

\section{A. Area Discretization}

In this subsection, we will demonstrate how to discretize the $2 \mathrm{D}$ area based on a piecewise constant approximation of $e(d)$.

To begin with, we use multiple piecewise constant segments $\varepsilon(d)$ to approximate the EMR function $e(d)$. We intelligently control the number of segments $K$ and their end points $\ell(1), \ldots, \ell(K)(\ell(0)=D, \ell(K)=D)$ such that the approximation error is less than a given small number $\epsilon$. After that, we draw concentric circles with radius $\ell(1), \ldots, \ell(K)$ for each charger, respectively. The approximated EMR from the charger at any point between adjacent circles should be uniform. Finally, the whole network plane is partitioned into multiple sub-area faces, which are shaped by these circles.

Taking Fig. 2 as an example. The EMR function $e(d)$ is approximated by 2 piecewise constant segments with endpoints $\ell(1)$ and $\ell(2)$. Then, given 3 chargers on the plane, we draw 2 concentric circles for each of them, and therefore, the network plane is partitioned into 13 sub-area faces, including the outer face with no EMR. For each sub-area face, such as $F_{q}$ in Fig. 2, the approximated EMR at any point within it from each charger should be constant.

We define the following approximation of EMR and bound its approximation error.

Definition 4.1: Setting $\ell(0)=0, \ell(K)=D$, and $\ell(k)=$ $\beta\left((1+\epsilon)^{k / 2}-1\right),(k=1, \ldots, K-1)$, the piecewise constant function $\varepsilon(d)$ can be defined as

$$
\varepsilon(d)=\left\{\begin{array}{l}
e(\ell(0)), d=\ell(0) \\
e(\ell(k-1)), \ell(k-1)<d \leq \ell(k)(k=1, \ldots K) \\
0, d>D
\end{array}\right.
$$

Note that $\epsilon$ is a given error threshold.

Theorem 4.1: With $\varepsilon(d)$, the approximation error of EMR is subject to

$$
\frac{\varepsilon(d)}{e(d)} \leq 1+\epsilon,(d \leq D)
$$


We proceed to bound the approximation error of EMR in each sub-area face, as well as the number of sub-area faces.

Let $\varepsilon(p)$ be the approximated EMR of any position $p$ in the face $F_{q}$, namely, $\varepsilon(p)=\sum_{i=1}^{n} \varepsilon_{q i} x_{i}, \forall p \in F_{q}$, where $\varepsilon_{q i}$ is a constant that denotes the approximated EMR stemming from the charger $s_{i}$ in the face $F_{q}$. Then, we have the following theorem.

Theorem 4.2: The approximation error of any position $p$ in the face $F_{q}$ satisfies

$$
\frac{\varepsilon(p)}{e(p)} \leq 1+\epsilon, \forall p \in F_{q} .
$$

Proof: Clearly, we have $\frac{\varepsilon(p)}{e(p)}=\frac{\sum_{i=1}^{n} \varepsilon_{q i} x_{i}}{\sum_{i=1}^{n} e\left(d\left(s_{i}, p\right)\right) x_{i}}$. Combining Eq. 6, the result follows.

Theorem 4.3: The number of sub-area faces $Z$ satisfies

$$
Z=O\left(N^{2} \epsilon^{-2}\right)
$$

Proof: By Def. 4.1, we can derive that the number of divided sub-areas $K$ is given by $K=\left\lceil\frac{\ln (e(0) / e(D))}{\ln (1+\epsilon)}\right\rceil$, which means $K=O\left(\epsilon^{-1}\right)$. Besides, it is clear that the number of all concentric circles is $N K$. Based on the classical results of [22], the number of sub-area faces formed by $N K$ circles satisfies $Z \leq(N K)^{2}-N K+2$. The result follows.

\section{B. Problem Reformulation}

After the approximation procedures introduced above, we reduce the number of constraints in $\mathbf{P 1}$ from infinite to finite. As a result, SCAPE is reformulated as

(P2)

$$
\begin{aligned}
\max & C_{1} \sum_{i=1}^{n}\left(\sum_{j=1}^{m} P\left(d\left(s_{i}, o_{j}\right)\right)\right) x_{i} \\
\text { s.t. } & C_{2} \sum_{i=1}^{n} P_{q i} x_{i} \leq R_{t},(q=1, \ldots, Z) \\
& 0 \leq x_{i} \leq 1,(i=1,2, \ldots, n) .
\end{aligned}
$$

This is a typical linear programming problem. Mathematically, it can be solved by integer programming solvers such as CPLEX [23]. However, the number of the faces will explode with a large network size and a small value of $\epsilon$. To tackle this challenge, it is desirable to develop distributed algorithms to address the problem. Before going into the details of distributed algorithms, we need to implement the area discretization method in a distributed manner.

\section{Distributed Implementation of Area Discretization}

Recall that chargers are assumed to know its location a priori. Each charger can exchange the location information with its neighbors, and thereby discretize its covered disk area accordingly. After that, each charger will obtain a list of linear constraints, and neighboring chargers must share at least one constraint as they have intersection sub-areas.

\section{Distributed Redundant Constraint Reduction}

In this section, we investigate how to identify and remove redundant linear constraints in a distributed way to reduce the computational effort. In particular, we propose a twostep algorithm to deal with this problem. We note that this

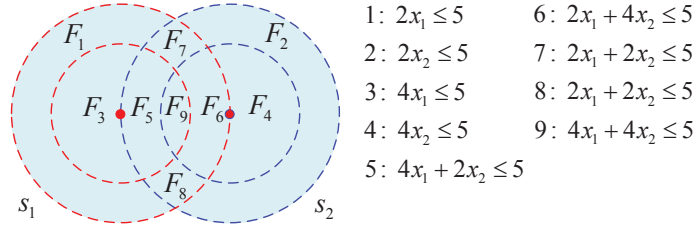

Fig. 3. Illustration of an example of constraint reduction

algorithm is only performed once after the deployment of chargers, and it doesn't need the knowledge of position or charging power of devices. Therefore, its computational time is amortized over time and thus is negligible.

By redundant constraints, we mean those constraints whose presence does not alter the optimum solution. We refer the reader to [24] for the formal definition of redundant constraints. As the existing algorithms for redundant constraint reduction, such as [25] and [26], are centralized and thus cannot be directly applied to our scenarios, we develop our own distributed algorithm inspired by [25] [26].

In our settings, each charger maintains a list of linear constraints which corresponds to the sub-area faces it covers, and neighboring chargers must share at least one linear constraint. In general, our goal is to collaboratively reduce the redundant constraints among chargers such that the number of the aggregated linear constraints (which merges the identical linear constraints between neighbors) is minimized.

Due to space limit, we just sketch our Distributed Redundant Constraint Reduction (DRR) algorithm. Basically, the distributed algorithm consists of two stages. The first stage is to locally remove trivial constraints which can be always satisfied. Then, the charger selectively collects neighboring linear constraints from neighbors which don't involve itself (a charger $s_{j}$ is said to be involved in a linear constraint if and only if the coefficient of $x_{j}$ in this constraint is positive), and employs linear programming method to further remove its redundant constraints. In particular, to identify the redundant constraints, the left-hand side of each constraint is optimized subject to the remaining constraints. The optimal value is then compared with the right-hand side value of the corresponding constraint to decide whether it is redundant or not.

Taking Fig. 3 as an example. The whole area is partitioned into 9 sub-area faces after area discretization for two chargers $s_{1}$ and $s_{2}$. Suppose the EMR threshold $R_{t}=5$, the approximated charging power in inner and outer sub-areas is 4 and 2 respectively. We thus obtain 9 linear constraints as listed in Fig. 3. At the first stage of DRR, the constraint 1, 2, 3, 4, 7 and 8 are identified as trivial constraints and removed at both chargers as they can always be satisfied.

Next, both chargers broadcast their constraints to each other. The charger $s_{1}$, for example, finds that all the received constraints involve itself, and therefore it neglects all of them. Next, for the rest constraints, such as the constraint 5 , it maximizes $4 x_{1}+2 x_{2}$, the left side of this constraint, subject to the constraints 6 and 9 . The optimal value is 4.5 , which is smaller than 5 . Therefore, the constraint 5 can be removed. So does the constraint 6 . Finally, only the constraint 9 remains. 


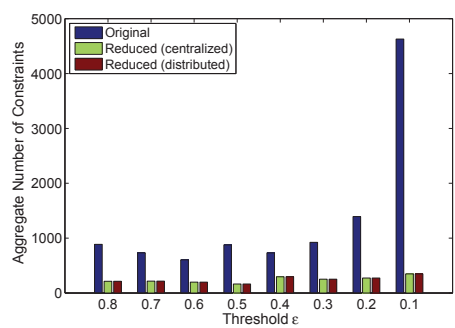

(a) Constraint Number vs. $\epsilon$

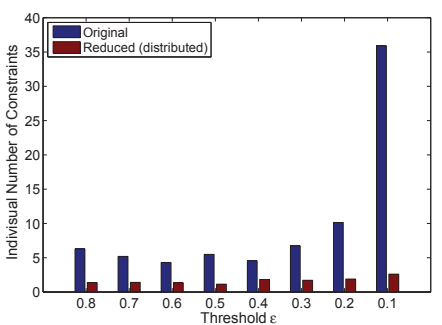

(b) Constraint Number vs. $\epsilon$

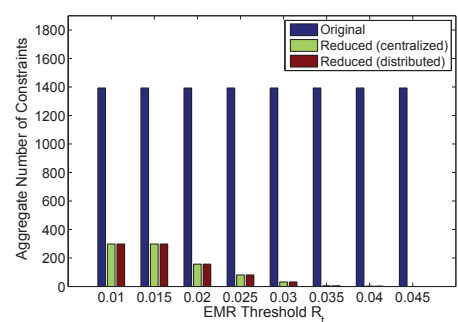

(c) Constraint Number vs. $R$

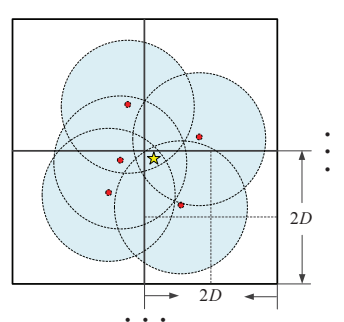

Fig. 5. Illustration of the coverage relationship

Fig. 4. Illustration of Redundant Constraint Reduction

We disclose the performance of the algorithm in the following theorem by comparing it to its centralized version, which conducts the two-step scheme based on the complete knowledge of all constraints coming from all chargers.

Theorem 5.1: The DRR algorithm achieves the same performance as the corresponding centralized version in that the aggregation of its obtained linear constraints is identical to that of the centralized algorithm.

Proof: We omit the details of the proof to save space.

Fig. 4 shows the performance of our DRR algorithm in terms of $\epsilon$ and $R_{t}$ by adopting the same parameter setting as that in Section VII-C1. In Fig. 4(a), the original aggregated number of constraints generally increases with a decreasing $\epsilon$, and rises to 4629 when $\epsilon=0.1$. In contrast, the number of reduced constraints of DRR algorithm is substantially smaller than the original number of constraints, e.g., it is only 348 when $\epsilon=0.1$, nearly $7.52 \%$ of the original one. Moreover, it is exactly equal to that produced by the corresponding centralized algorithm. Further, Fig. 4(b) shows that the average number of constraints possessed by individual chargers is nearly proportional to the aggregated number of constraints.

Besides, Fig. 4(c) shows that the number of reduced constraints of DRR algorithm or centralized algorithm decreases steadily when $R_{t}$ becomes larger, and drops to 0 when $R_{t}=0.045$ since $R_{t}$ is even bigger than the maximum possible EMR over the plane. In contrast, the original number of constraints remains unchanged.

\section{VI. $(1-\epsilon)$-Approximation Distributed Algorithm}

In this section, we discuss how to develop a $(1-\epsilon)$ approximation algorithm.

To begin with, we divide the whole area into uniform squares with size $2 D * 2 D$, where $D$ is the disk radius of chargers' coverage area. Since each charger is aware of its location, it can easily classify itself into a specific regular square given a global reference anchor point. Apparently, by applying this partition method, the chargers in non-adjacent squares will not have their coverage areas intersected. By "adjacent", we mean two squares share at least one end point. Fig. 5 shows an example where four squares are adjacent to each other.

As shown in Fig. 6, we group $m * m$ squares into a larger grid, which we call $m$-grid for short. For simplicity, suppose that the whole area can be divided into an integral number of $m$-grid (if it is not the case, we can add phantom squares to

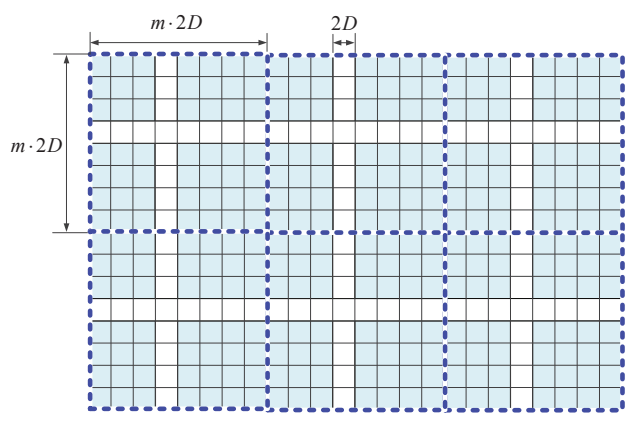

Fig. 6. Illustration of overall partition

achieve this goal). In Fig. 6, there are 6 m-grids enclosed by blue dotted boundaries after grouping.

To decompose $\mathbf{P 2}$ into minor ones and solve them in a distributed manner, we can selectively turn off some chargers such that the entire area can be separated into several subareas. Fig. 6 shows an example that the chargers located in those white strips are switched off, then the whole area is repartitioned into 12 sub-areas, each of which contains at most $m * m$ squares. Specifically, we require that each $m$-grid adopt the same select-and-turn-off policy, namely, turning off all the chargers located at the $i$-th row and the $j$-th column of the $m$-grid. We use a two-tuple $\langle i, j\rangle$ to denote such policy. Fig. 7 demonstrates two different policies, $\langle 4,4\rangle$ and $\langle 6,6\rangle$. By the policy $\langle 4,4\rangle$, for example, all the chargers at the 4 -th row and the 4 -th column should be turned off. Fig. 6 illustrates the ultimate result when each $m$-grid adopts the policy $\langle 4,4\rangle$.

Consequently, in each partitioned sub-area, such as those 12 sub-areas in Fig. 6, we can apply a local linear programming method to determine the powers of the chargers inside independently of other sub-areas. This is because the nearest distance between any sub-areas is at least $2 D$, which is sufficient to avoid the influence of EMR from chargers in other sub-areas. If no confusion arises, we call these newly formed sub-areas as new m-grids for simplicity.

So far, our problem has been boiled down to two subproblems, namely, how to determine the size of an m-grid (i.e., $m$ ) and how to determine the select-and-turn-off policy adopted in each m-grid. In general, for the first subproblem, we will prove that $m$ only relates to the error threshold $\epsilon$, and has nothing to do with the present device distribution. In contrast, the second subproblem should be addressed based on the knowledge of device locations and their charging utility. 

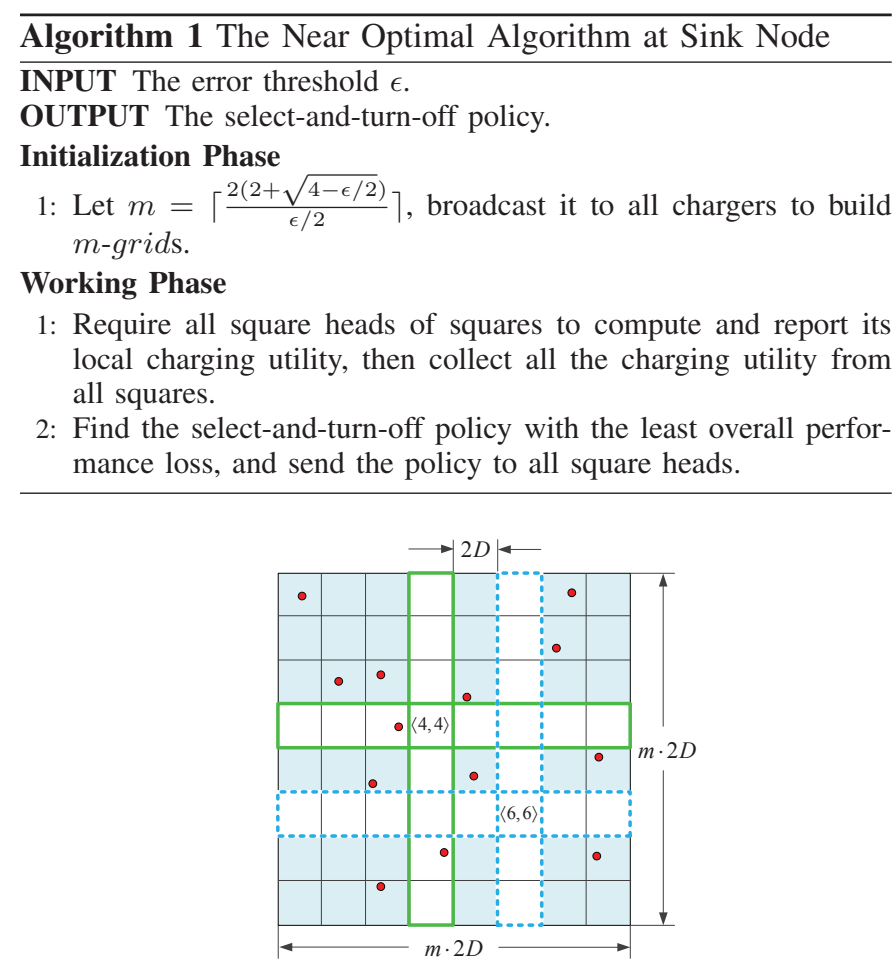

Fig. 7. Illustration of $m$-grid

In particular, we establish a sink node to collect the charging utility of all squares, find the select-and-turn-off policy with the least overall performance loss, and disseminate the final solution to all squares. By intelligently choosing the policy with the least performance loss, we can achieve a factor of $(1-\epsilon)$ of the optimum.

We present the details of the near optimal algorithms performed at the sink node and at the square heads, which serves as an agent between the sink node and the devices in their square, in Alg. 1 and Alg. 2, respectively. In general, in the initialization phase, the sink node decides the size of $m$-grids, namely $m=\left\lceil\frac{2(2+\sqrt{4-\epsilon / 2})}{\epsilon / 2}\right\rceil$, and broadcasts them to all chargers, which construct m-grids accordingly. We will prove that such a value of $m$ contributes to achieving $(1-\epsilon)$ approximation ratio. Note that linear constraint extraction and redundant constraint reduction, together with square partition, have already been done before this procedure at square heads in all squares.

Next, the working phase mainly includes two stages. At the first stage, the sink node collects all necessary information from all square heads, and thereby determines the select-andturn-off policy with the least overall performance loss and sends it out. For the second stage, after receiving the policy, each square head reassigns itself to a new m-grid. And in each new m-grid, a head is elected to facilitate the local computation of the linear programming problem.

Note that the distributed algorithm conducted in chargers that are not square nodes is simply to report information to square heads, receive command and adjust power accordingly.

Theorem 6.1 reveals the performance of the distributed algorithm.

Theorem 6.1: The distributed algorithm achieves $(1-\epsilon)$ approximation ratio.
Algorithm 2 The Near Optimal Algorithm at Square Heads

INPUT The location, the maximum received powers of its covered devices.

OUTPUT The objective power.

\section{Initialization Phase}

1: Use area discretization technique with error threshold $\epsilon / 2$ to extract linear constraints, then apply DRR algorithm to reduce redundant constraints.

2: Classify itself into a specific square, and elect a local square head.

3: Receive the parameter $m$ from the sink node, and classify itself into a $m$-grid.

\section{Working Phase}

1: When receiving the request from the sink node to report local charging utility, disseminate the request to other chargers in this square, and collect all the information from them.

2: Compute the local optimal solution in this square, send it to the sink node.

3: Receive the command from the sink node. If the command is to turn off the chargers in this square, disseminate the command to each charger in this square, turn itself off. Otherwise, assign itself to a new m-grid according to the received command, and elect a local head of the $m$-grid.

4: if It is the m-grid head then

5: Disseminate the request for information to other square heads in this $m$-grid, and collect all the information from them.

6: $\quad$ Compute the local optimal solution in this m-grid, send it to square heads in this $m$-grid. Adjust its power according to the optimal solution.

7: else

8: When receiving the request from the $m$-grid head to report the related information, send it to the $m$-grid head.

9: $\quad$ Receive the command from the $m$-grid head, disseminate it to all chargers in the square, and adjust its power according to the command.

10: end if

Proof: We prove this theorem in the Appendix for a better flow of the paper.

We remark that, the election of m-grid heads at Step 3 in Alg. 2 can be done in the initialization phase to save the real time computational cost and reduce delay. This calls for enumeration for all possible $m^{2}$ number of formation of new $m$-grids in advance, and the space to store computed results.

Moreover, Alg. 2 can be adapted to be fully distributed by removing the notion of sink node, square head and m-grid head, and letting chargers exchange information with their neighbors round by round until all the required information is collected, and then computing the optimal solution. Such an adapted algorithm is, apparently, more robust, but is more computationally demanding.

Suppose the charger deployment density in the field is bounded, then for typical charger networks we have the following theorem. Note that $n$ is the number of chargers.

Theorem 6.2: The number of execution rounds of the near optimal algorithm is $O(\log n)$.

\section{Simulation Results}

In this section, we first present simulation results to evaluate our proposed algorithms in terms of error threshold $\epsilon$, charger number, EMR threshold $R_{t}$ and delay. After that, we give 

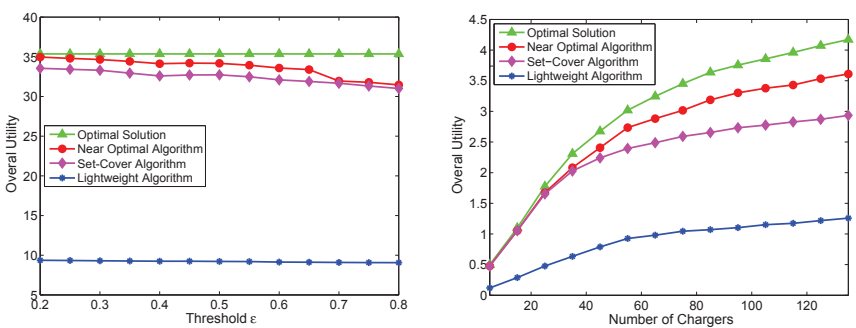

Fig. 8. Overall Utility vs. Threshold Fig. 9. Number

insights of how the distribution of chargers affects the overall charging utility.

\section{A. Evaluation Setup}

Unless otherwise specified, we use the following evaluation settings. Assume there are 400 chargers and $10^{4}$ devices uniformly distributed over a $1000 m * 1000 m$ 2D square area. Moreover, we set $\alpha=100, \beta=100$ and $D=20$ for the charging model, $C_{1}=1$ for the EMR model and $C_{2}=0.001$ for the utility model. The error threshold is $\epsilon=0.4$, and the EMR threshold is $R_{t}=0.018$. Moreover, every point on the simulation curves stands for the average value of 100 instances with different random seeds and device deployments.

\section{B. Baseline Setup}

As currently there is no algorithm available for safe charging with adjustable power, we introduce two algorithms for comparison. The first algorithm borrows the idea of SetCover. After collecting the information from all chargers, this algorithm greedily picks the charger which covers the maximum number of devices, and adjusts its power as large as possible while assuring the EMR safety, until no further addition of chargers is possible considering the EMR safety. Then, it broadcasts the results to all chargers.

The second algorithm is a lightweight algorithm. In this algorithm, each square head simply gathers the information locally in the square, computes the optimal solution, and then cuts down the power of each charger in the solution to $1 / 4$. The obtained solution must satisfy the EMR safety requirement by similar analysis in the proof to Theorem 6.1.

In addition, we approximate the optimal solution by partitioning the area in a fine-grained way and solving the obtained linear programming problem in a centralized way.

\section{Performance Comparison}

1) Impact of Threshold $\epsilon$ : We first investigate the influence of the error threshold $\epsilon$ on the overall utility. As depicted in Fig. 8, the overall utility of the optimal solution remains unchanged and is equal to 35.4 as $\epsilon$ grows, while that of the other three algorithms decreases steadily. Specifically, our near optimal solution is always larger than $(1-\epsilon)$ of the optimal value, and outperforms that of the Set-Cover algorithm by nearly $4 \%$ on average. Such small performance gap between these two algorithms is due to the fact that the charger distribution in this case is relatively sparse and most chargers are isolated from others, and therefore, the outputs of the
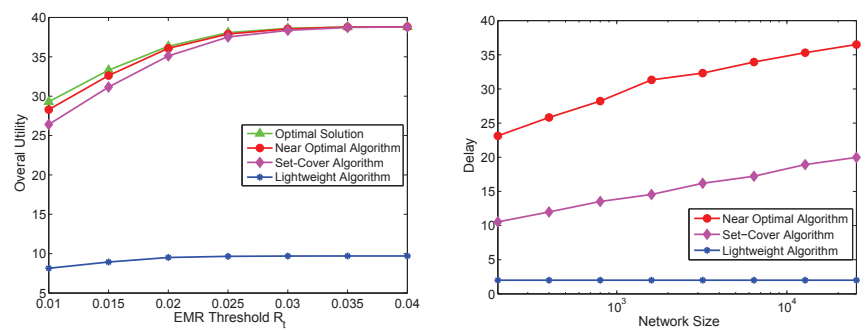

Fig. 10.
Threshold $R_{t}$

two algorithms are very similar. The lightweight algorithm performs the worst because of the huge potential utility loss when it cuts down the computed power to $1 / 4$.

Further, we observe that the achieved utility of the near optimal solution experiences a drop when $\epsilon=0.7$. This is because the specified size of $m$-grid is bigger than the network size when $\epsilon<0.7$, and thus there is no need for m-grid partition, and no further performance loss will occur. The case is the opposite for $\epsilon \geq 0.7$.

2) Impact of Charger Number: We proceed to examine the influence of the charger number on the overall utility. To reduce the computational time, we reduce the field size to $200 m * 200 m$ and the number of devices to 400 , and set $\epsilon=0.8$. In Fig. 9, it can be seen that the performance gap between the optimal algorithm and the near optimal solution, as well as the Set-Cover algorithm, is pretty small when the number of chargers is small. This is because with a sparse distribution, most of the chargers can be set to its maximum power without hurt the EMR safety for all these three algorithms. Nevertheless, the gaps between the three algorithms become conspicuous with more chargers deployed. The near optimal solution has an performance gain of up to $23.0 \%$ over the Set-Cover algorithm, and its performance loss compared with the optimal solution is no more than $13.5 \%$, much less than $80 \%$ allowed by Theorem 6.1. Again, the light weight algorithm has the worst performance.

3) Impact of EMR Threshold $R_{t}$ : We are also interested in the impact of the EMR threshold $R_{t}$ on the overall utility. As illustrated in Fig. 10, not surprisingly, the overall utility of all algorithms grows with an increasing $R_{t}$. Note that we set $\epsilon=0.2$. The near optimal algorithm also outperforms the Set-Cover algorithm. Overall, the optimal solution is nearly $0.7 \%$ higher than that of our near optimal algorithm, which in turn enjoys a performance gain of up to $7.2 \%$ over the Set-Cover algorithm. Furthermore, when $R_{t}$ exceeds 0.035, all the charger can be tuned to its maximum power while guaranteeing the EMR safety, and thereby the overall utility of the optimal, near optimal and Set-Cover algorithms reach the maximum value 38.8 . Similarly, the lightweight algorithm keeps the utility value of 9.7 when $R_{t}$ exceeds 0.025 .

4) Impact of Network Size on Delay: We study the impact of network size on the overall delay in this part. We fix the density of chargers to be $1 / 400$, set $\epsilon=0.4$, and let the communication radius be twice the charging radius, i.e., 40. In Fig. 11, we can see that the delay of the lightweight algorithm 


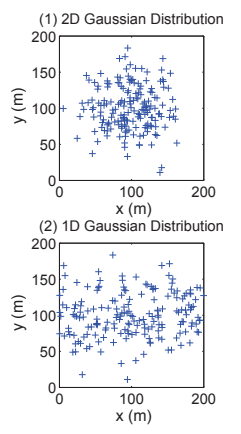

(a)

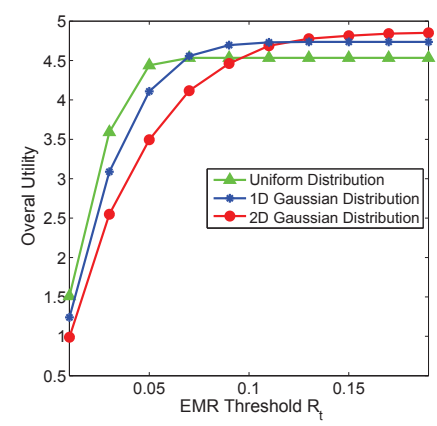

(b)
Fig. 12. Illustration of non-uniform distributions and results

keeps constant, since it only requires local communication within a square. In contrast, the delay of the Set-Cover algorithm increases nearly proportional to the logarithm of the network size, which is mainly due to the algorithm's data collection and solution dissemination processes.

For the near optimal algorithm, the slope of the curve is twice that of the Set-Cover algorithm when the number of chargers is smaller than 1600 , and is the same otherwise. This is because the size of $m$-grids is precisely 1600 . Therefore, if the number of chargers exceeds 1600 , since the number of required computation steps in a m-grid is constant, the increment of delay only depends on the first stage of data collection and dissemination process.

\section{Insights}

In this section, we provide insights of how the distribution of chargers affects the overall charging utility. Suppose there are 50 chargers deployed in a $200 m * 200 m$ field, and 400 devices uniformly distributed in the same field. We consider three kinds of distributions, i.e., uniform distribution, 2D Gaussian distribution and 1D Gaussian distribution. By 2D Gaussian distribution, both of the $\mathrm{x}$-coordinate and $\mathrm{y}$-coordinate of a charger are randomly selected from a Gaussian distribution with $\mu=100$ and $\sigma=100 / 3$. Compared with 2D Gaussian distribution, the only difference of 1D Gaussian distribution is that its X-coordinate is uniformly selected among [0 200]. Fig. 12(a) illustrates examples of them.

As shown in Fig. 12(b), all the utility of three distributions first increases rapidly and then smoothly, and finally becomes constant since all the chargers are adjusted to their maximum power. An interesting observation is that the utility achieved for the uniform distribution is first superior to that corresponding to the other two distributions, and soon becomes inferior to them. This is because when $R_{t}$ is small, the chargers with uniform distribution have larger average distance between each other, and thus are allowed to tune their power to a greater value while assuring EMR safety, which results in a higher utility. In contrast, with a large $R_{t}$, the chargers are able to adopt a larger power, even the maximum power, without violating the EMR safety, and therefore, the number of covered devices becomes the dominant factor affecting the overall utility. Note that, there are a number of chargers located near the boundaries of the field in the uniform distribution case, whose charging area inevitably covers the region outside the field. This coverage waste thus leads to a loss of the overall utility. In contrast, the chargers for the other two distributions are more concentrated, and thus results in a smaller coverage waste as well as a larger overall utility.

Furthermore, for the same reasons, the performance for the 1D Gaussian distribution case is below that of the 2D Gaussian distribution given a large $R_{t}$.

\section{FIELD EXPERIMENTS}

In this section, we build testbed and conduct field experiments to verify our theoretical findings.

\section{A. Testbed}

Generally, we use the same testbed as that in [19]. The testbed consists of 8 TX91501 power transmitters produced by Powercast [3] [27], and 2 rechargeable sensor nodes. We place the chargers on the vertices and middle points of edges of a $2.4 m * 2.4 m$ square area, and one wireless rechargeable sensor node at the center of the square area, and the other to the right side of the first one with distance $0.4 \mathrm{~m}$, as illustrated in Fig. 13. Also shown in this figure are a laptop and an AP connecting to it. The AP is responsible to collect the information of received power from the sensor nodes, and then send it to the laptop.

Note that TX91501 power transmitters are actually directional, whose charging region can be modeled as a sector with angle $60^{\circ}$ and radius 4 . We tune the orientations of charger 1 to 8 as $296.56^{\circ}, 296.56^{\circ}, 243.44^{\circ}, 26.56^{\circ}, 153.44^{\circ}, 63.44^{\circ}$, $116.56^{\circ}$ and $116.56^{\circ}$, respectively. Apart from this, as the power of TX91501 power transmitters is not adjustable, we put a piece of copper foil tape with proper length and width in front of chargers, and adjust its position and bending angle such that the received power and EMR at locations further than the tape are nearly uniformly cut down to a desired level.

\section{B. Adapted Algorithm and Comparison Algorithm}

In consideration of the charging features of the TX91501 power transmitters, we make necessary modifications to our algorithms to accommodate this case. We omit the details to save space. Second, we let the two sensors sample the charging power from each charger at the beginning of the algorithm to reduce the model error and make our algorithm robust to environmental variation. We then perform our algorithms based on the sampled values.

We compare our algorithm to the SCP algorithm, a centralized algorithm proposed in [19] that addresses the similar problem, except that the power of chargers is assumed to be not adjustable.

\section{Experimental Results}

As shown in Fig. 14, we compare the utility computed based on sampling value with real utility under three different values of $R_{t}$ for both SCAPE and SCP algorithms. Note that the charging utility is the summation of that of the two sensor nodes. It can be observed that the computed utility 


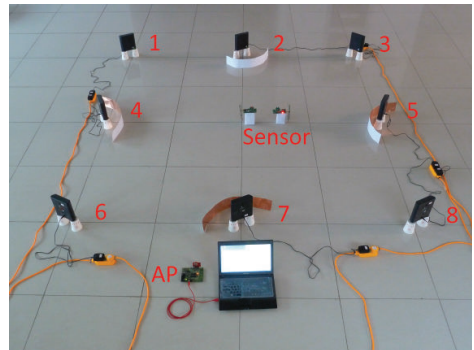

Fig. 13. Illustration of field experiment

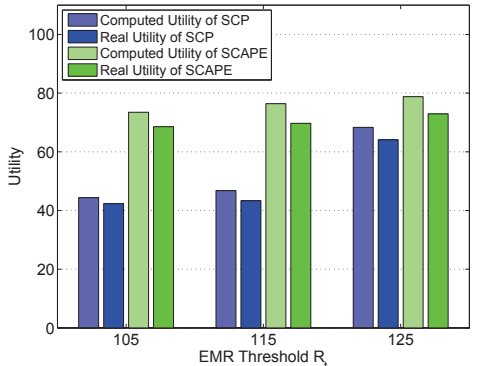

Fig. 14. Utility vs. EMR Threshold $R_{t}$

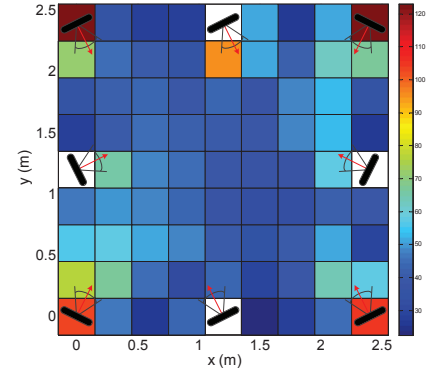

Fig. 15. An example of EMR distribution of both algorithms is always larger than the real utility, but the performance gap is no more than $8.7 \%$. Besides, when $R_{t}$ decreases from 125 to 105 , the achieved real utility of the SCP algorithm drops by $33.9 \%$, while that of the SCAPE algorithm reduces only $6.0 \%$. On average, the SCAPE algorithm is $41.1 \%$ better than the SCP algorithm.

We set $R_{t}$ as $125 \mu \mathrm{W} / \mathrm{cm}^{2}$, compute the adjusting factors for the charger 1 to 8 , i.e., $1,0.60,1,0.72,0.78,1,0.75$ and 1 , and put pieces of copper foil tape in front of the charger 2, 4, 5 and 7 correspondingly. We measure the EMR values at $9 * 9$ grid points of the square region except the locations of charger $2,4,5$ and 7 since the power there is not properly adjusted. We plot the results in Fig. 15, and observe that the EMR peaks at the location of charger 1 and is equal to $122 \mu \mathrm{W} / \mathrm{cm}^{2}$, which is less than $R_{t}$. This fact validates the correctness of our SCAPE algorithm. Note that, though the EMR value at the locations of the charger 2, 4, 5 is missed, it can be deduced that they won't exceed $R_{t}$ too by the surrounding EMR values.

\section{CONCLUSION}

In this paper, we have studied the problem of how to adjust the power of chargers to maximize the overall charging utility while guaranteeing the EMR safety. We employed novel techniques to reformulate the problem and further reduce its computational efforts. We then presented a distributed algorithm with approximation ratio $(1-\epsilon)$ to address the problem. Finally, we conducted both extensive simulations and field experiments to validate our theoretical findings.

Due to its distributed nature and consideration of computational effort throughout the design process, our proposed scheme could be easily incorporated into real systems. In our future work, we will take other practical concerns into consideration, such as fairness of charging.

\section{ACKNOWLEDGMENT}

This work is supported by National 973 project of China under Grants No.2012CB316201 and No.2014CB340303, National Natural Science Foundation of China under Grants No.61133006, No.61321491, No.61373130, No.61170247 and No.613230428, National Key Project of China under Grant No.2013ZX01033002-003, Research and Innovation Project for College Graduate Students of Jiangsu Province in 2012 under Grant No.CXZZ12_0056, and the Shanghai Recruitment Program of Global Experts.

\section{REFERENCES}

[1] A. Kurs et al., "Wireless power transfer via strongly coupled magnetic resonances," science, vol. 317, no. 5834, pp. 83-86, 2007.

[2] "http://www.seattle.intel-research.net/wisp/."

[3] "www.powercastco.com."

[4] "www.powermat.com."

[5] "http://www.laptopmag.com/reviews/laptops/dell-latitude-3330.aspx."

[6] "http://evworld.com/news.cfm?newsid=24420."

[7] "http://www.friendsofcrc.ca/projects/sharp/sharp.html."

[8] S. He et al., "Energy provisioning in wireless rechargeable sensor networks," in INFOCOM, 2011, pp. 2006-2014.

[9] H. Dai et al., "Impact of mobility on energy provisioning in wireless rechargeable sensor networks," in WCNC, 2013.

[10] T.-C. Chiu et al., "Mobility-aware charger deployment for wireless rechargeable sensor networks," in APNOMS, 2012.

[11] J.-H. Liao, "Optimized charger deployment for wireless rechargeable sensor networks," 2013.

[12] R. P. Wicaksono et al., "Wireless Grid: Enabling Ubiquitous Sensor Networks with Wireless Energy Supply," pp. 1-5, 2011.

[13] H. Dai et al., "Quality of energy provisioning for wireless power transfer,' IEEE Transactions on Parallel and Distributed Systems, 2014.

[14] M. P. Ntzouni et al., "Transient and cumulative memory impairments induced by gsm $1.8 \mathrm{ghz}$ cell phone signal in a mouse model," Electromagnetic biology and medicine, 2013.

[15] M. OLTEANU et al., "Dangerous temperature increase from em radiation around metallic implants," 2012.

[16] M. Havas et al., "Provocation study using heart rate variability shows microwave radiation from $2.4 \mathrm{ghz}$ cordless phone affects autonomic nervous system," European Journal of Oncology Library, vol. 5, 2010.

[17] M. Edwards et al., "Effects of heat on embryos and foetuses." International journal of hyperthermia: the official journal of European Society for Hyperthermic Oncology, North American Hyperthermia Group, vol. 19, no. 3, pp. 295-324, 2002.

[18] O. P. Gandhi et al., "Exposure limits: The underestimation of absorbed cell phone radiation, especially in children," Electromagnetic Biology and Medicine, vol. 31, no. 1, pp. 34-51, 2012.

[19] H. Dai et al., "Safe charging for wireless power transfer," in INFOCOM, 2014.

[20] J. Xu, L. Liu, and R. Zhang, "Multiuser miso beamforming for simultaneous wireless information and power transfer," arXiv preprint arXiv:1303.1911, 2013.

[21] K. Huang and E. G. Larsson, "Simultaneous information and power transfer for broadband wireless systems," IEEE Transactions on Signal Processing, vol. 61, no. 23, pp. 5972-5986, 2012.

[22] M. De Berg et al., Computational geometry: algorithms and applications. Springer, 2008.

[23] E. D. Dolan and J. J. More, "Benchmarking optimization software with performance profiles," Mathematical programming, vol. 91, no. 2, pp. 201-213, 2002

[24] S. Paulraj and P. Sumathi, "A comparative study of redundant constraints identification methods in linear programming problems," Mathematical Problems in Engineering, vol. 2010, 2010.

[25] A. Brearley et al., "Analysis of mathematical programming problems prior to applying the simplex algorithm," Mathematical programming, vol. 8, no. 1, pp. 54-83, 1975.

[26] R. Caron et al., "A degenerate extreme point strategy for the classification of linear constraints as redundant or necessary," Journal of Optimization Theory and Applications, vol. 62, no. 2, pp. 225-237, 1989.

[27] H. Dai et al., "Near optimal charging and scheduling scheme for stochastic event capture with rechargeable sensors," in MASS, 2013. 


\section{APPENDIX}

\section{A. Proof of Theorem 6.1}

Proof: Denote by $g_{i j}^{k}$ the square located at the $i$-th row and $j$-th column of the $k$-th $m$-grid. At Step 2 of Working Phase in Alg. 2, each square $g_{i j}^{k}$ computes the optimal local charging utility, which we denote by $u_{i j}^{k}$. Let $\tilde{U}^{*}$ be the optimal overall charging utility for the problem P2, it is easy to see

$$
\sum_{k} \sum_{j} \sum_{i} u_{i j}^{k} \geq \tilde{U}^{*}
$$

Obviously, there are $m^{2}$ different select-and-turn-off policies for all $m$-grids. For a given policy $\langle i, j\rangle$, the overall performance loss is

$$
L_{i j}^{k}=\sum_{i^{\prime}=i} u_{i^{\prime} j^{\prime}}^{k}+\sum_{j^{\prime}=j} u_{i^{\prime} j^{\prime}}^{k}-u_{i j}^{k} .
$$

Therefore, the overall performance loss of the network is

$$
\mathcal{L}_{i j}=\sum_{k} L_{i j}^{k}=\sum_{k}\left(\sum_{i^{\prime}=i} u_{i^{\prime} j^{\prime}}^{k}+\sum_{j^{\prime}=j} u_{i^{\prime} j^{\prime}}^{k}-u_{i j}^{k}\right) .
$$

Next, we investigate the summation of the performance loss for all possible select-and-turn-off strategies, which can be formally expressed as

$$
\begin{aligned}
\sum_{i} \sum_{j} \mathcal{L}_{i j} & =\sum_{i} \sum_{j}\left(\sum_{k}\left(\sum_{i^{\prime}=i} u_{i^{\prime} j^{\prime}}^{k}+\sum_{j^{\prime}=j} u_{i^{\prime} j^{\prime}}^{k}-u_{i j}^{k}\right)\right) \\
& =(2 m-1) \sum_{k} \sum_{j} \sum_{i} u_{i j}^{k} .
\end{aligned}
$$

Subsequently, we intend to disclose the relationship between $\sum_{k} \sum_{j} \sum_{i} u_{i j}^{k}$ and $\tilde{U}^{*}$. We first present the following simple yet important observation.

Observation A.1: Any point in the field can only be covered by chargers from at most 4 squares.

Fig. 5 illustrates an example of this observation. When a point, marked in a yellow star in the figure, located in the upleft corner of a square, it can only be covered by the chargers from this square, along with those from the adjacent three squares as depicted.

Based on Observation A.1, we suppose the EMR at a point $p$ consists of four parts, i.e., $\varepsilon_{1}, \varepsilon_{2}, \varepsilon_{3}$ and $\varepsilon_{4}$ from chargers in 4 adjacent squares $\left(\varepsilon_{i}=0\right.$ if there is no EMR from that square). Obviously, we have $\varepsilon_{1} \leq R_{t}$, $\varepsilon_{2} \leq R_{t}, \varepsilon_{3} \leq R_{t}$ and $\varepsilon_{4} \leq R_{t}$. As a result, if we reduce the power of each charger in the field to $1 / 4$, the corresponding aggregate EMR at $p$ satisfies $\varepsilon(p)=1 / 4 *\left(\varepsilon_{1}+\varepsilon_{2}+\varepsilon_{3}+\varepsilon_{4}\right) \leq R_{t}$, which means the reduced power is a feasible solution. Consequently, the overall charging utility becomes $1 / 4 * \sum_{k} \sum_{j} \sum_{i} u_{i j}^{k}$ after power reduction. Since $\tilde{U}^{*}$ is optimal, we have

$$
1 / 4 * \sum_{k} \sum_{j} \sum_{i} u_{i j}^{k} \leq \tilde{U}^{*} .
$$

Combining Eq. 13 and Eq. 14 gives

$$
\sum_{i} \sum_{j} \mathcal{L}_{i j} \leq 4(2 m-1) \tilde{U}^{*}
$$

Finally, according to the pigeonhole principle, we claim that there must exist a policy $\langle i, j\rangle$ that has the performance loss

$$
\mathcal{L}_{i j} \leq \frac{4(2 m-1)}{m^{2}} \tilde{U}^{*}
$$

In practice, we can compute all the possible value of $\mathcal{L}_{i j}$ and pick out the least one, then it must conform to Inequality 16. This is done at Step 2 of Working Phase in Alg. 1.

After obtaining the least $\mathcal{L}_{i j}$ and thereby determining the policy $\langle i, j\rangle$, the sink sends the policy to the whole network. Chargers acting as square heads received the command either turns off itself as shown at Step 3 of Working Phase in Alg. 2 for square heads, or participates in electing $m$-grid head and performing a local linear programming algorithm within a new $m$-grid. Suppose the summation of the obtained charging utility of all newly formed $m$-grids is $U$. It is obvious that

$$
U+\mathcal{L}_{i j} \geq \tilde{U}^{*} .
$$

Combining Eq. 16 and Eq. 17, we have

$$
U \geq\left(1-\frac{4(2 m-1)}{m^{2}}\right) \tilde{U}^{*} .
$$

Given an arbitrarily small value of $\epsilon$, we set

$$
m=\left\lceil\frac{2(2+\sqrt{4-\epsilon / 2})}{\epsilon / 2}\right\rceil,
$$

as at Step 1 of Initialization Phase in Alg. 1, we then have

$$
U \geq(1-\epsilon / 2) \tilde{U}^{*} .
$$

Further, let $U^{*}$ be the optimal overall charging utility of the original problem P1. Suppose that the adjusting factor for the charger $s_{i}$ is $x_{i}^{*}$ in the optimal solution. We then have

$$
C_{2} \sum_{i=1}^{n} P\left(d\left(s_{i}, p\right)\right) x_{i}^{*} \leq R_{t}
$$

for any point $p$.

Suppose the point $p$ is inside the face $F_{q}$. By Eq. 7, the approximated EMR at $p$ after area disretization satisfies

$$
\varepsilon(p)=C_{2} \sum_{i=1}^{n} P_{q i} x_{i}^{*} \leq(1+\epsilon / 2) C_{2} \sum_{i=1}^{n} P\left(d\left(s_{i}, p\right)\right) x_{i}^{*} .
$$

Note that we set the error threshold for area discretization to $\epsilon / 2$. It immediately follows

$$
C_{2} \sum_{i=1}^{n} P_{q i} \frac{x_{i}^{*}}{(1+\epsilon / 2)} \leq C_{2} \sum_{i=1}^{n} P\left(d\left(s_{i}, p\right)\right) x_{i}^{*} \leq R_{t},
$$

which implies $\frac{x_{i}^{*}}{(1+\epsilon)}$ is a feasible solution for the problem $\mathbf{P 2}$.

Since $\tilde{U}^{*}$ is the optimal solution for $\mathbf{P 2}$, we thus have

$$
\begin{aligned}
\tilde{U}^{*} & \geq C_{1} \sum_{i=1}^{n}\left(\sum_{j=1}^{m} P\left(d\left(s_{i}, o_{j}\right)\right)\right) \frac{x_{i}^{*}}{(1+\epsilon / 2)} \\
& \geq \frac{1}{(1+\epsilon / 2)} C_{1} \sum_{i=1}^{n}\left(\sum_{j=1}^{m} P\left(d\left(s_{i}, o_{j}\right)\right)\right) x_{i}^{*} \\
& \geq \frac{1}{(1+\epsilon / 2)} U^{*} \geq(1-\epsilon / 2) U^{*} .
\end{aligned}
$$

Combining Eqs. 20 and 21, we have

$$
U \geq(1-\epsilon / 2) *(1-\epsilon / 2) U^{*} \geq(1-\epsilon) U^{*} .
$$

This completes the proof. 